\title{
Using population synthesis of massive stars to study the interstellar medium near OB associations
}

\author{
R. $\operatorname{Voss}^{1,2}$, R. Diehl ${ }^{1}$, D. H. Hartmann ${ }^{3}$, M. Cerviño ${ }^{4}$, J. S. Vink ${ }^{5}$, G. Meynet $^{6}$, M. Limongi ${ }^{7}$, and A. Chieffi ${ }^{7}$ \\ 1 Max-Planck-Institut für extraterrestrische Physik, Giessenbachstrasse, 85748, Garching, Germany \\ e-mail: rvoss@mpe.mpg.de \\ 2 Excellence Cluster Universe, Technische Universität München, Boltzmannstr 2, 85748, Garching, Germany \\ 3 Department of Physics and Astronomy, Clemson University, Kinard Lab of Physics, Clemson, SC 29634-0978, USA \\ 4 Instituto de Astrofísica de Andalucía (CSIC), Camino bajo de Huétor 50, Apdo. 3004, Granada 18080, Spain \\ 5 Armagh Observatory, College Hill, Armagh, BT61 9DG, Northern Ireland, UK \\ ${ }^{6}$ Geneva University, Geneva Observatory, 1290 Versoix, Switzerland \\ INAF Osservatorio Astronomico di Roma, via Frascati 33, 00040 Monteporzio Catone Roma, Italy
}

Received 2 April 2009 / Accepted 7 July 2009

\section{ABSTRACT}

\begin{abstract}
Aims. We study the massive stars in $\mathrm{OB}$ associations and their surrounding interstellar medium environment, using a population synthesis code.

Methods. We developed a new population synthesis code for groups of massive stars, where we model the emission of different forms of energy and matter from the stars of the association. In particular, the ejection of the two radioactive isotopes ${ }^{26} \mathrm{Al}$ and ${ }^{60} \mathrm{Fe}$ is followed, as well as the emission of hydrogen ionizing photons, and the kinetic energy of the stellar winds and supernova explosions. We investigate various alternative astrophysical inputs and the resulting output sensitivities, especially effects due to the inclusion of rotation in stellar models. As the aim of the code is the application to relatively small populations of massive stars, special care is taken to address their statistical properties. Our code incorporates both analytical statistical methods applicable to small populations, as well as extensive Monte Carlo simulations.

Results. We find that the inclusion of rotation in the stellar models has a large impact on the interactions between OB associations and their surrounding interstellar medium. The emission of ${ }^{26} \mathrm{Al}$ in the stellar winds is strongly enhanced, compared to non-rotating models with the same mass-loss prescription. This compensates the recent reductions in the estimates of mass-loss rates of massive stars due to the effects of clumping. Despite the lower mass-loss rates, the power of the winds is actually enhanced for rotating stellar models. The supernova power (kinetic energy of their ejecta) is decreased due to longer lifetimes of rotating stars, and therefore the wind power dominates over supernova power for the first $6 \mathrm{Myr}$ after a burst of star-formation. For populations typical of nearby star-forming regions, the statistical uncertainties are large and clearly non-Gaussian.
\end{abstract}

Key words. stars: winds, outflows - stars: early-type - stars: abundances - ISM: abundances - gamma rays: observations

\section{Introduction}

Massive stars dynamically shape the interstellar medium around them on timescales of a few Myr (see e.g. Lozinskaya 2001). Due to their high mass loss rates (Castor et al. 1975; Lamers \& Morton 1976; Barlow et al. 1981) and terminal velocities of their winds (Howarth \& Prinja 1989; Lamers et al. 1995) and their supernova explosions (Jones 1998) they are important sources of mechanical power causing interstellar medium (ISM) turbulence and the formation of shells and cavities (e.g. van der Hucht 1987; Leitherer et al. 1992; Maeder \& Conti 1994). Their large UV luminosities, they furthermore cause the surrounding medium to be photoionized to significant distances (Panagia 1973; Vacca et al. 1996).

The mixing of ejecta from young stars into the interstellar medium is an important process in the interplay between star formation and galaxy evolution. A unique window into these processes is provided by the radioactive isotope ${ }^{26} \mathrm{Al}$, traced by its $\gamma$-ray decay line at $1808.63 \mathrm{keV}$. With a mean lifetime of $\sim 1 \mathrm{Myr}$ it is a long-term tracer of nucleosynthesis for populations of sources able to eject it sufficiently fast after synthesis (Prantzos $\&$ Diehl 1996). The COMPTEL instrument aboard the Compton
Gamma Ray Observatory (CGRO) has mapped the ${ }^{26} \mathrm{Al}$ emission at $1809 \mathrm{keV}$ in a 9-year full sky survey. From a comparison between the image morphology and the known spiral arm tangents and regions of star formation, one can deduce that massive stars dominate the Galactic ${ }^{26} \mathrm{Al}$ production, and that the contribution from novae and AGB stars must be relatively minor (Diehl et al. 1995; Knödlseder et al. 1999; Plüschke 2001; Prantzos \& Diehl 1996; Diehl et al. 2006). Localized groups of massive stars in star-forming regions such as Cygnus and Orion emit clear ${ }^{26} \mathrm{Al}$ signals, and these regions provide an important contribution to the total amount of ${ }^{26} \mathrm{Al}$ present in the Milky Way, and it is therefore important to understand these basic building blocks. Typically, massive stars eject a few $10^{-5} M_{\odot}$ of ${ }^{26} \mathrm{Al}$ through their winds and supernova (SN) explosions (see e.g. Limongi \& Chieffi 2006), and the total mass of ${ }^{26} \mathrm{Al}$ in the Milky Way is estimated to be $2.8 \pm 0.8 M_{\odot}$ (Diehl et al. 2006). A similar tracer is the isotope ${ }^{60} \mathrm{Fe}$, observed by its $1173 \mathrm{keV}$ and $1333 \mathrm{keV}$ decay lines (Smith 2004; Harris et al. 2005). This isotope is also emitted in the supernova explosions (Limongi \& Chieffi 2006). The lifetime of ${ }^{60} \mathrm{Fe}$ has recently been revised to $\sim 3.8 \mathrm{Myr}$, from the previous estimate of $\sim 2 \mathrm{Myr}$ (Rugel, private communication). While the lines are significantly weaker than 
Table 1. Parameter names used throughout the paper.

\begin{tabular}{lll}
\hline \hline Parameter name & Parameter description & References \\
\hline geneva05 & stellar evolution with rotation & Meynet \& Maeder (2005); Palacios et al. (2005) \\
geneva05alt & stellar evolution with rotation & Meynet \& Maeder (2005) \\
geneva97 & stellar evolution & Maeder \& Meynet (1994); Meynet et al. (1997) \\
LC06 & stellar evolution & Limongi \& Chieffi (2006) \\
wind08 & wind velocities & Lamers et al. (1995); Niedzielski \& Skorzynski (2002) \\
wind00 & wind velocities & Howarth \& Prinja (1989); Prinja et al. (1990) \\
yieldsLC06 & supernova nucleosynthesis yields & Limongi \& Chieffi (2006) \\
yieldsWW95 & supernova nucleosynthesis yields & Woosley et al. (1995); Cerviño et al. (2000a) \\
atmosMS & stellar atmospheres & Kurucz (1992); Martins et al. (2005); Smith et al. (2005) \\
atmosSmith & stellar atmospheres & Kurucz (1992); Smith et al. (2005) \\
atmos0LD & stellar atmospheres & Kurucz (1992); Schmutz et al. (1992); Schaerer \& de Koter (1997) \\
\hline
\end{tabular}

the ${ }^{26} \mathrm{Al}$ line, it is possible to detect them when integrating over large regions of the Milky Way.

We have developed a population synthesis code that follows the evolution of massive stars and computes the ejection of ${ }^{26} \mathrm{Al}$ and ${ }^{60} \mathrm{Fe}$ from a star-forming region. To facilitate studies of the dynamics of the surrounding environment, the code also computes the kinetic energy and the mass ejected from the winds and the SN explosions, as well as the ionizing flux. In contrast to Starburst99 (Leitherer et al. 1999; Vazquez \& Leitherer 2005), we focus on the study of relatively small, nearby starforming regions in the Milky Way. Due to the limited number of massive stars in thsee individual regions, stochastic processes can cause their behaviour to deviate strongly from the mean results obtained for large populations. Because of this, the code is designed to not only calculate the average behaviour from an analytical integration over the initial mass function (IMF), but also the expected distribution of output quantities for finite populations. This is both done using analytical approximations and Monte Carlo simulations. To do this we implemented the statistical methods of Cerviño \& Luridiana (2006). These enable us to determine when Monte Carlo simulations are mandatory in order to properly describe the distributions, and how many simulations are needed to obtain statistically robust results.

Here we describe the population synthesis code, and discuss the dependence on the various physical input models. We compare our results to the predictions of Starburst 99 for kinetic luminosity and UV flux, and to Cerviño et al. (2000a) for radioactive isotope production. We discuss the simulated stellar populations in the context of the inter-stellar medium (ISM), with emphasis on the changes induced by recent stellar evolution tracks for massive stars (Limongi \& Chieffi 2006; Meynet \& Maeder 2005).

\section{Population synthesis of massive stars}

The synthesis code calculates time profiles of the emission of radioactive elements ${ }^{26} \mathrm{Al}$ and ${ }^{60} \mathrm{Fe}$ from a population of massive stars, for an assumed star formation history. The code also traces the energy and mass ejected from the stars through stellar winds and supernova explosions, as well as the UV radiation they emit. These quantities are the primary tools necessary to study the dynamical effects of the interaction between massive stars and the surrounding ISM. We interpolate between the discrete inputs from the models, mainly using linear interpolation, except for temperatures, masses, luminosities, surface gravities and stellar ages, which we interpolate logarithmically, unless otherwise noted.

The code is divided into two separate parts: in the first one stellar tracks are used to calculate time profiles for individual stars, and we interpolate between these to create a fine grid of stellar tracks as a function of progenitor mass. For a given time we tabulate the stellar properties as a function of initial stellar mass, thereby creating isochrones. The second part of the code uses the isochrones as input, and for each isochrone calculates integrated quantities for a given population of stars, weighting the isochrone values by the IMF. This part of the program calculates analytically the mean value of the distribution of properties we are interested in, as well as some high order moments that allows an analytical estimation of the probability distribution of populations with a finite (and small) size (see Cerviño \& Luridiana 2006, for details). It also estimates these distributions with Monte Carlo simulations for populations of any size, where intial stellar masses are chosen randomly, with relative probabilities according to the assumed IMF. These outputs are complementary in the sense that the average values are suitable for very large populations of stars, such as entire galaxies, whereas the Monte Carlo simulations are necessary for very small star-forming regions. The analytical probability distributions are useful in intermediate cases ranging from about one hundred to thousands of massive stars. As we aim to model star forming regions in the solar neighbourhood, we restricted our study to stellar models of solar metallicity.

The details of the physical ingredients of the code are described in the following. For a number of processes, switches are included to select between alternative models. We identify these through convenient parameter names that are used throughout the paper when discussing the outputs of the code. The paramer names are summarized in Table 1.

\subsection{Stellar evolution}

For the stellar evolution of massive stars several alternative sets of stellar tracks are implemented. The default (geneva05) consists of the solar metallicity, rotating stellar models described in Meynet \& Maeder (2005) and Palacios et al. (2005). These models all have ZAMS rotation velocities of $300 \mathrm{~km} \mathrm{~s}^{-1}$, producing time averaged equatorial velocities on the main sequence between 200 and $250 \mathrm{~km} \mathrm{~s}^{-1}$. There are two versions of these models, one including the calculation of ${ }^{26} \mathrm{Al}$ (Palacios et al. 2005) for stars with initial masses between 25 and $120 M_{\odot}$, and one without (Meynet \& Maeder 2005) for stars with initial masses between 9 and $120 M_{\odot}$. We therefore use the stellar tracks of Palacios et al. (2005) above $25 M_{\odot}$, combined with the models of Meynet \& Maeder (2005) below this limit as our default. However, to enable the calculation of ${ }^{26} \mathrm{Al}$, parts of the numerical calculation was changed between the two sets of models (Meynet, private communication). We therefore test our results on the stellar energy ejection against those obtained using 
Table 2. Classification criteria for WR stars, and their wind velocities.

\begin{tabular}{lccc}
\hline \hline WR type & Surface abundances & Wind velocity (wind08) & Wind velocity (wind00) \\
\hline WNL & $0.4>\mathrm{H}_{\mathrm{s}}>0.1$ & 1250 & 1900 \\
WNE & $\mathrm{H}_{\mathrm{s}}<0.1 \& \mathrm{C}_{\mathrm{s}} / \mathrm{N}_{\mathrm{s}}<10$ & 2000 & 1650 \\
WC6-9 & $\mathrm{H}_{\mathrm{s}}<0.1 \& \mathrm{C}_{\mathrm{s}} / \mathrm{N}_{\mathrm{s}}>10 \&\left(\mathrm{C}_{\mathrm{s}}+\mathrm{O}_{\mathrm{s}}\right) / \mathrm{He}_{\mathrm{s}}<0.5$ & 1760 & 1810 \\
WC4-5 & $\mathrm{H}_{\mathrm{s}}<0.1 \& \mathrm{C}_{\mathrm{s}} / \mathrm{N}_{\mathrm{s}}>10 \&\left(\mathrm{C}_{\mathrm{s}}+\mathrm{O}_{\mathrm{s}}\right) / \mathrm{He}_{\mathrm{s}}<1.0$ & 2650 & 2820 \\
WO & $\mathrm{H}_{\mathrm{s}}<0.1 \& \mathrm{C}_{\mathrm{s}} / \mathrm{N}_{\mathrm{s}}>10 \&\left(\mathrm{C}_{\mathrm{s}}+\mathrm{O}_{\mathrm{s}}\right) / \mathrm{He}_{\mathrm{s}}>1.0$ & 3000 & 3000 \\
\hline
\end{tabular}

${ }^{*} \mathrm{H}_{\mathrm{s}}, \mathrm{C}_{\mathrm{s}}, \mathrm{N}_{\mathrm{s}}$ and $\mathrm{He}_{\mathrm{s}}$ are the fractional surface abundances (mass fraction) of hydrogen, carbon, nitrogen and helium, respectively, and the wind velocities are in $\mathrm{km} \mathrm{s}^{-1}$.

stellar tracks of Meynet \& Maeder (2005) only (geneva05alt), see Sect. 3.3.

The main effects of rotation on the yields of ${ }^{26} \mathrm{Al}$, and the energy and mass ejection are the following (Meynet \& Maeder 2005; Palacios et al. 2005): Rotational mixing allows surface enrichment in ${ }^{26} \mathrm{Al}$ at an earlier evolutionary stage than obtained in models without rotational mixing. When rotation is not accounted for, surface ${ }^{26} \mathrm{Al}$ enrichment occurs only when the deep layers of the stars where ${ }^{26} \mathrm{Al}$ is synthesized are uncovered by the stellar winds. In rotating models rotational diffusion enables surface enrichments well before the regions processed by hydrogen burning are uncovered by the stellar winds. This effect increases the quantity of ejected ${ }^{26} \mathrm{Al}$ with respect to non-rotating models. For the same reasons rotating stars present surface abundance characteristics of the Wolf-Rayet stages before the stellar winds have uncovered the core. This causes the stars to enter the WR-phase earlier and to increase the WR lifetimes. For example a $40(85) M_{\odot}$ non-rotating star has a WR lifetime of merely $\sim 0.1 \mathrm{Myr}$, whereas the rotating counterpart has a WR lifetime of almost 0.4 (1.4) Myr. Also rotational mixing enables stars with masses as low as $22 M_{\odot}$ to become WR-stars. These effects allow rotating models to eject more ${ }^{26} \mathrm{Al}$ into the ISM than nonrotating ones. This also increases the mass loss and therefore the amount of kinetic energy ejected into the ISM. Furthermore stellar lifetimes are increased by rotation, with a $15-25 \%$ increase in the hydrogen burning lifetimes. This delays the onset of the supernova explosions, and lowers the supernova rate for a population of rotating stars, compared to a population of non-rotating stars.

Rotational mixing can in many respects improve the correspondence between the outputs of stellar models and the observed features of massive stars. For instance, rotational mixing may be the cause for surface nitrogen enrichments in OB main sequence stars (see e.g. Hunter et al. 2007; Maeder et al. 2008). With the relatively low mass loss rates presently favoured (see below), non-rotating models underpredict the observed ratio of WR to O-type stars. Rotational mixing favors WR formation, and rotating models are thus in better agreement with observations. Provided that most of the nitrogen enriched stars and WR stars are produced by single star evolution, rotation appears as a key physical ingredient of the models. However, we note that the comparison of models with observations and the discussion about whether the rotating models reproduce the observed properties of massive stars better than non-rotating models is currently a subject of debate (e.g. Hamann et al. 2006; Meynet \& Maeder 2005; Eldridge \& Vink 2006; Vazquez \& Leitherer 2007).

Two alternative sets of non-rotating models are implemented. One set (geneva97) consists of the solar metallicity models of Meynet et al. (1997) combined with the models of Maeder \& Meynet (1994) for stars below $25 M_{\odot}$. The other set (LCQ6) consists of the stellar tracks of Limongi \& Chieffi (2006).
The main differences between the sets of stellar evolution models relevant to our study are the inclusion of rotation in the geneva05 stellar models, and the updated wind mass-loss estimates included in the geneva05 and LCO6 calculations. These new estimates reduce the wind mass-loss rates of the geneva 05 and the LCO6 models significantly compared to the geneva97 models: in the Wolf-Rayet (WR) phases the inclusion of clumping in the interpretation of the observational data leads to reduced mass-loss rates by a factor of 2-3 compared to the earlier models (Nugis \& Lamers 2000). Also the theoretical mass-loss rates for O and B stars of Vink et al. (2000, 2001) are lower by similar factors in comparison to earlier empirical values of e.g. de Jager et al. (1988). See the recent review by Puls et al. (2008) for an extensive description of mass-loss rates.

We follow each stellar track through the provided evolutionary points and calculate the quantities needed for the population synthesis.

\subsubsection{Kinetic energy}

The rate of kinetic energy emitted in the winds of the stars is calculated from $E_{\mathrm{k}}=1 / 2 \dot{M} v_{\infty}^{2}$, where $\dot{M}$ is the mass-loss rate from the stellar atmosphere and $v_{\infty}$ is the velocity of the wind at infinity (the terminal velocity). This represents the energy available once the wind has escaped the gravitational potential of the star. The interaction of the winds with the ISM can convert a large fraction of the energy into gas turbulence and radiation. The mass loss rate is given by the stellar tracks. To calculate the wind velocity we first coarsely classify the stars according to the following criteria (Leitherer et al. 1999): stars with a mass loss rate above $\dot{M}=10^{-3.5} M_{\odot} \mathrm{yr}^{-1}$ and effective temperatures in the range $3.75>\log T_{\text {eff }}<4.4$ are classified as luminous blue variables (LBVs). Stars with an effective temperature above $\log T_{\text {eff }}=4.0$ and a fractional abundance of hydrogen at the surface below 0.4 , are considered WR-stars. They are furthermore divided into subclasses according to the surface abundances (Smith \& Maeder 1991; Leitherer et al. 1999), see Table 2.

The terminal velocity of the wind depends on the type of star. We utilize two different prescriptions for the calculation: in the default mode (wind08) stars outside the categories defined above are divided into hot stars with $v_{\infty}=2.6 v_{\text {esc }}$ and cool stars with $v_{\infty}=1.3 v_{\text {esc }}$ at a temperature of $\log T_{\text {eff }}=4.32$ (Lamers et al. 1995), where $v_{\text {esc }}$ is the escape velocity at the surface of the star. Luminous blue variables are assumed to have winds with low velocities $\left(200 \mathrm{~km} \mathrm{~s}^{-1}\right)$ and the velocities of the WR-winds are estimated from Niedzielski \& Skorzynski (2002) and given in Table 2.

The alternative mode wind 00 corresponds to the assumptions in Starburst 99 (Leitherer et al. 1992). Here stars outside the categories have wind velocities given by $v_{\infty}=0.85 \times(0.58+$ $\left.2.04 \times \log \frac{R}{R_{\odot}}\right) \times v_{\text {esc }}$ (Howarth \& Prinja 1989), luminous blue 
variables again have $v_{\infty}=200 \mathrm{~km} \mathrm{~s}^{-1}$, and the velocities of the WR-winds are estimated from Prinja et al. (1990), and given in Table 2.

\subsubsection{Radioactive isotopes}

The wind ejection of the radioactive isotope ${ }^{26} \mathrm{Al}$ is followed along the stellar tracks. The ejection rate is found from multiplying the surface abundance fraction of ${ }^{26} \mathrm{Al}$ with the mass loss rate, and the amount of ${ }^{26} \mathrm{Al}$ present in the interstellar medium around the star is calculated by taking into account the decay timescale of $\sim 1$ Myr. The other radioactive isotope considered in this study, ${ }^{60} \mathrm{Fe}$, is not ejected in the stellar winds, but only in the supernova explosions. For this isotope we consider two decay timescales, with $\sim 2 \mathrm{Myr}$ as the default and $~ 3.8 \mathrm{Myr}$ suggested by recent measurements. At the last point of the stellar tracks the radioactive yields from the supernova explosions are added. We have two prescriptions for calculating the yields: in the default mode (yieldsLC2006) we use the yields calculated by Limongi \& Chieffi (2006). The yields are found based on the initial mass of the stars, interpolating linearly between their data points. In the alternative method (yieldsWW95) the yields below initial masses of $25 M_{\odot}$ are taken from Woosley $\&$ Weaver (1995). Above this mass we set the output of each star to $5.0 \times 10^{-5} M_{\odot}$ for both ${ }^{26} \mathrm{Al}$ and ${ }^{60} \mathrm{Fe}$. This is approximately the average yields found in Cerviño et al. (2000a) from linking the core mass of the geneva97 stellar tracks with the supernova yields of Woosley et al. (1995). We note that in Cerviño et al. (2000a), the results depend strongly on the method used (see their Figs. 1 and 2). Our crude approximation therefore reflects unknown physics. The total yield is not affected dramatically by these uncertainties, as there are few massive stars, and for ${ }^{26} \mathrm{Al}$, the emission from these stars is dominated by the wind contribution.

\subsection{Creation of the stellar isochrones}

We use the stellar tracks to create isochrones for a user-defined set of times after the onset of star formation. To facilitate the interpolation between the stellar tracks, for each of the tracks a subset of 51 equivalent evolutionary points are extracted according to the definitions given in Maeder (1990). The reduction of the stellar tracks to 51 points eliminates some of the finer details of stellar evolution, but the uncertainties due to this simplification are small compared to the uncertainties of defining equivalent evolutionary points and interpolating between stellar models with different initial mass. For each isochrone an array of initial stellar masses is defined, and stellar tracks are produced by interpolation between the equivalent points of the nearest input stellar tracks. We note that the ages at the equivalent points are interpolated using a cubic spline in logarithmic space, as lower degree interpolation methods create artificial results (e.g. in the supernova rate, see Cerviño et al. 2001, Fig. 1). The isochrone values are then found by interpolation along these stellar tracks. We note that the geneva 05 tracks are only calculated to the end of central helium burning, while the geneva97 tracks are followed to the end of central carbon burning. We use the last available grid points and extrapolate it towards the end, thus neglecting the stages between the ends of the stellar tracks and the supernova explosions.

\subsection{Calculation of population properties}

The stellar isochrones are the basis of our population synthesis. Each isochrone is used to calculate the population properties, both by integrating the isochrone analytically and using Monte Carlo techniques. Both methods sample the number of stars of a given mass according to the initial mass function (IMF), for which we use the Salpeter mass function (Salpeter 1955) as the default, with $8 M_{\odot}$ and $120 M_{\odot}$ as lower and upper integration limits. As only massive stars are investigated here, we do not consider more complicated IMF models (e.g. Scalo 1986; Kroupa 2001) that also address the shape of the mass function at lower masses. We furthermore include an implementation of the statistical methods described in Cerviño \& Luridiana (2006) to account for statistical variability of stellarmass weightings for smaller numbers of stars. This allows us to calculate analytically the statistical distribution of the output quantities, depending on the number of stars in the stellar population, similar to what can be done with Monte Carlo simulations. We do not include the effect of binaries (Vanbeveren et al. 2007; Eldridge et al. 2008). The frequency of interacting binaries, and the effects of the interactions are poorly known, and the effects on the parameters studied in this paper are difficult to assess. Such interactions might remove outer layers of primary stars, and rejuvenate secondary stars. Both processes can potentially enhance the ${ }^{26} \mathrm{Al}$ ejection (Langer et al. 1998).

\subsubsection{Supernova explosions}

In this study, we assume that the kinetic energy ejected in a supernova explosion is $10^{51} \mathrm{erg} \mathrm{s}^{-1}$ (e.g. Woosley et al. 1995), irrespective of the progenitor mass. The supernova rate is derived as an average over a period of $0.1 \mathrm{Myr}$ and from this the "kinetic luminosity" of the supernovae is calculated. The mass ejected in supernova explosions is found from the difference between the mass of the last point in the stellar evolution code, and the mass of the compact remnant. We assume that all neutron stars have a canonical mass of $1.4 M_{\odot}$ and that all black holes have a mass of 7.0 $M_{\odot}$. The supernova explosions of stars with initial masses below $25 M_{\odot}$ are assumed to produce neutron stars whereas stars above this mass limit are assumed to produce black holes. Our results are not sensitive to these values within reasonable ranges. For example, if the most massive stars end up as neutron stars due to mass loss, this will only change the mass of the ejected matter by $\sim 5-10 \%$, as the majority of the mass is ejected in the winds at earlier evolutionary stages. It should be noted that the geneva97 and geneva05 stellar tracks do not cover the final stages of stellar evolution, and the mass ejected in the supernova explosions may therefore be overestimated. However, the mass loss from stellar winds is underpredicted by the same amount, and these effects should cancel out to some extent.

\subsubsection{Ionizing flux}

The ionizing flux of each star is calculated by linking stellar atmosphere models to the state of the star. The surface gravity and the effective temperature are used to find the atmosphere model with the closest resemblance. In the WR-phases, we use the method of Smith et al. (2005) to find these values at an optical depth of $\tau=10$. In this method, the effective temperature is simply given by $T_{*}=0.6 \cdot T_{\mathrm{hyd}}+0.4 \cdot T_{2 / 3}$, where $T_{\mathrm{hyd}}$ is the hydrostatic effective temperature of the stellar models, and $T_{2 / 3}$ is the corrected surface temperature given in the geneva97 and geneva05 stellar models. This parameter is unavailable in the 
L\&CQ5 stellar models, and the ionizing flux is therefore not calculated for these.

The default set of atmosphere models (atmosMS) applied for OB stars (Martins et al. 2005) and for WR stars (Smith et al. 2005) both take non-LTE, wind and line-blanketing effects into account. For stars with parameters beyond the range of these models we use Kurucz (1992) atmospheres. For comparison with Starburst99 we include an option of using the models of (Smith et al. 2005) for both WR and OB stars (atmosSmith), and for comparison with earlier studies an alternative set of models (atmosOLD) consisting of the WR models of Schmutz et al. (1992) and the OB models of Schaerer \& de Koter (1997), again supplemented by the models of Kurucz (1992).

\section{Population synthesis results}

We combine the results obtained from integrating over the individual isochrones to create time profiles of the average ejection of ${ }^{26} \mathrm{Al},{ }^{60} \mathrm{Fe}$, kinetic energy, hot gas and the ionizing flux. In this section we discuss these individual quantities, and their dependence on the assumptions made.

\subsection{Comparison with previous studies}

Synthesis of stellar populations is an important tool for understanding stellar clusters and galaxies. Since the first studies (Tinsley 1978; Bruzual 1983) the sophistication of the models have increased drastically (e.g. Bruzual \& Charlot 2003). The majority of these models are designed to predict the spectra of large stellar populations over a wide range of ages and metallicities, for which the exact parameters of the few massive stars are not of major importance. This is quite different from the goal of our study, which is the calculation of parameters of interest for the interactions between massive stars and their environment. Most studies are therefore not suited for comparison with the present study. Thus we use Starburst99 for comparing our results on the ionizing flux and kinetic luminosity. For this purpose we produce a model with parameters similar to parameters of their studies. We choose a model with a Salpeter IMF (Salpeter 1955), between 8-120 $M_{\odot}$ with the parameters geneva $97^{1}$, wind 00 , atmosSmith and yieldsWW95. The comparison for a cluster with a total mass of $10^{6} M_{\odot}$ in the given mass range are shown in Fig. 1. There is obviously good agreement between both models. The few discrepancies (e.g. the somewhat larger wind power between 2 and $3 \mathrm{Myr}$ in our model) can all be traced back to different interpolation schemes, and therefore represent unknown physics due to the limited stellar grid. The only previously published population synthesis of ${ }^{26} \mathrm{Al}$ and ${ }^{60} \mathrm{Fe}$ is Cerviño et al. (2000a). Again we find good agreement with their results. Only the early supernova ejection of ${ }^{26} \mathrm{Al}$ is higher in our model. This is due to the fact that their supernova yields decrease for the highest mass stars, whereas for our parameter yieldsWW95 they are constant above $25 M_{\odot}$.

\subsection{Radioactive isotopes}

Compared to the previous population synthesis study of ${ }^{26} \mathrm{Al}$ and ${ }^{60} \mathrm{Fe}$ (Cerviño et al. 2000a), our study includes new stellar models, specifically geneva05 and LCO6. Both take the reduced

\footnotetext{
1 While the geneva05alt stellar models are included in Starburst99 (Vazquez \& Leitherer 2007), this part of the code is not publicly available yet.
}

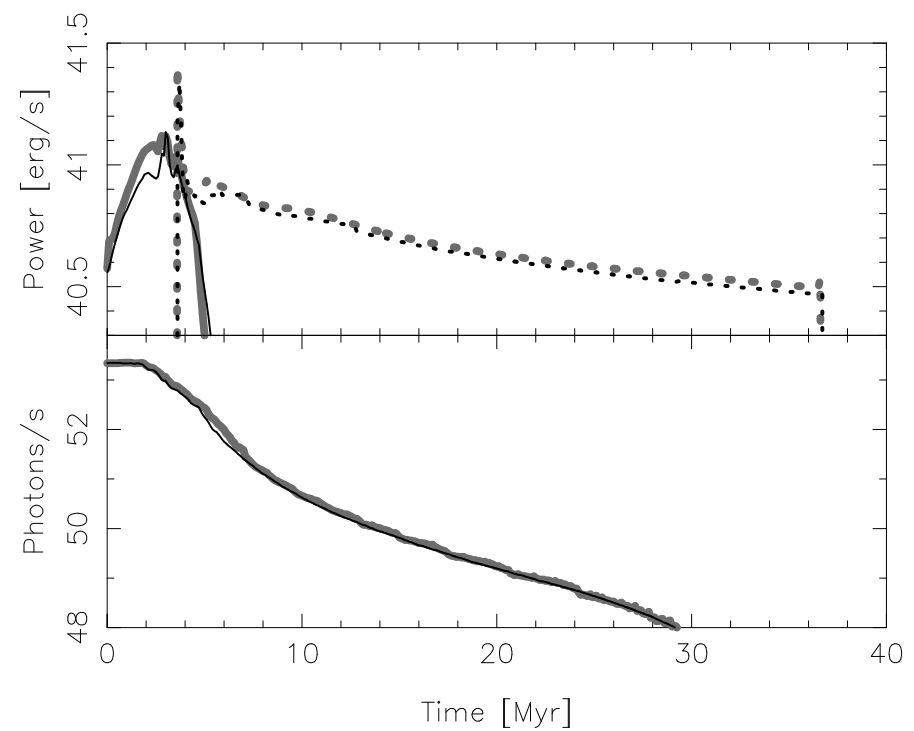

Fig. 1. Comparison between Starburst99 (thin black lines) and our results (thick grey lines) for a co-eval population. top: time profiles of the kinetic power emitted through the stellar winds (solid) and supernova explosions (dotted). Bottom: the ionizing flux.

mass loss estimates into account, and the geneva 05 model furthermore includes the effects of stellar rotation. The LCO6 model consistently includes the supernova explosions of the very massive stars. While these improvements have been discussed for individual stars and for the overall ejection from a population of stars (Palacios et al. 2005; Limongi \& Chieffi 2006), the effects on the time profile have not been evaluated in detail. The time profiles of ${ }^{26} \mathrm{Al}$ and ${ }^{60} \mathrm{Fe}$ in the interstellar medium for a coeval population of stars are shown in Fig. 2. The ${ }^{26} \mathrm{Al}$ has been divided into the wind and supernova contributions. Only the supernova contribution to ${ }^{60} \mathrm{Fe}$ is shown, as the wind contribution is negligible.

The wind contribution of ${ }^{26} \mathrm{Al}$ is relatively similar for the geneva05 and the geneva97 models. This is quite surprising, given the relatively large differences between these models. The reason is that the lower wind mass-loss in the geneva 05 models is compensated by two effects. One is the rotational mixing of elements, which leads to larger surface abundances. The other is the longer lifetime of the rotating models, which causes the amount of material lost to be similar to the amount lost in the geneva97 model, despite the lower rate of mass loss. This is also the reason that the ${ }^{26} \mathrm{Al}$ time profile shows a more extended peak for the rotating models. The LCO6 models, on the other hand, predict much less ${ }^{26} \mathrm{Al}$ ejection by the pre-supernova wind, for two main reasons: with the reduced mass loss, the rate of ejection is low, and the ${ }^{26} \mathrm{Al}$ is not effectively mixed to the surface.

For the supernova ejections the differences between the model predictions of ${ }^{26} \mathrm{Al}$ and ${ }^{60} \mathrm{Fe}$ are smaller, with the main effect coming from the longer lifetime of the rotating models causing a slightly less peaked profile. On the other hand, drastic differences between the predictions for the first $10 \mathrm{Myr}$ are obvious when comparing yieldsWW95 and yieldsLC2006. This probably mainly reflects the uncertainties in the final structure of the very massive stars, at the time of the supernova explosions. The low mass-loss in the models of (Limongi \& Chieffi 2006) gives very massive cores, that produce large amounts of the elements. The higher mass-loss rates assumed in the study of Cerviño et al. (2000a) gives lighter cores, and this results in the yieldsWW95 being lower than the yieldsLC2006. We note that 


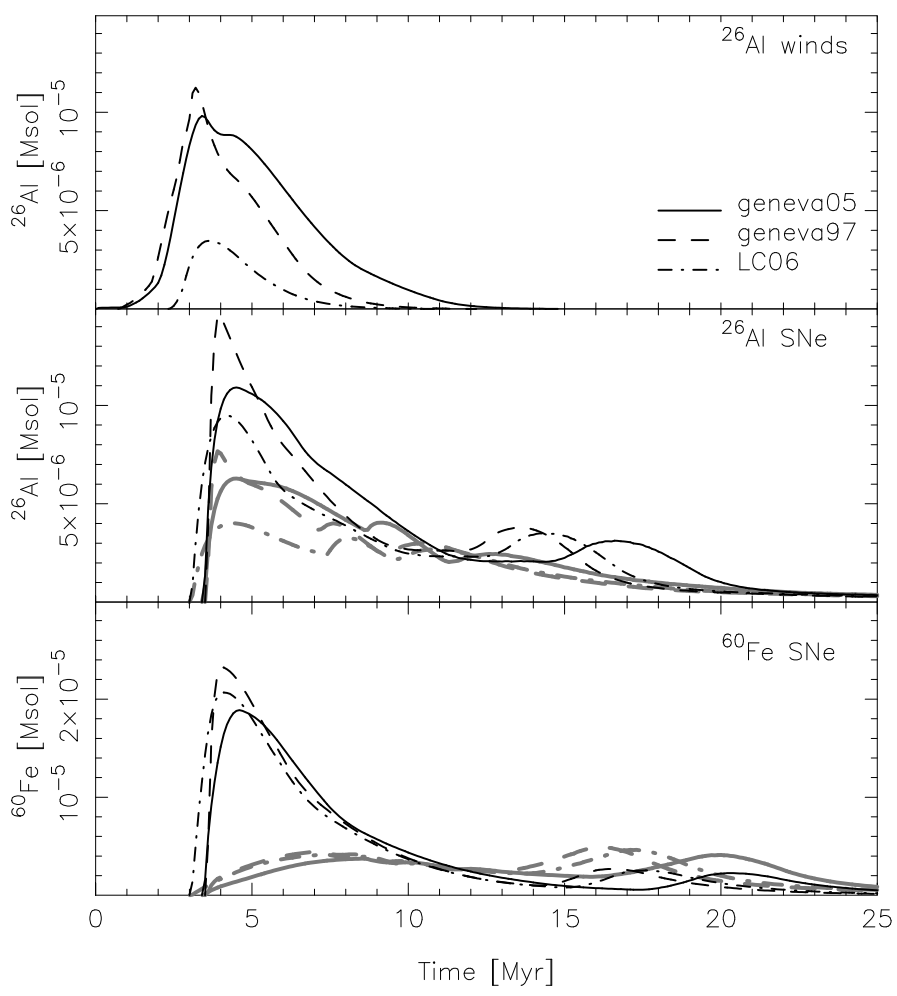

Fig. 2. Average time profiles of ${ }^{26} \mathrm{Al}$ and ${ }^{60} \mathrm{Fe}$ for a coeval population of stars, for the three different sets of stellar models available. The lines show the amount of the elements present in the ISM per star in the 8-120 $M_{\odot}$ range. The ${ }^{26} \mathrm{Al}$ yields are divided between the wind and the supernova contributions, whereas the wind contribution of ${ }^{60} \mathrm{Fe}$ is negligible. The black lines indicate the results using the yieldsLC2006 supernova yields, whereas the grey lines indicates the results using the yieldsWW95 yields.

with the very large theoretical uncertainties, observations of ${ }^{26} \mathrm{Al}$ and ${ }^{60} \mathrm{Fe}$ thus have the potential to place interesting constraints on the final evolutionary state of very heavy stars, although in an indirect way, since only the integrated effect of different sources can be observed.

In Fig. 3, the average time profiles of ${ }^{26} \mathrm{Al}$ (including both the wind and the supernova contributions) and ${ }^{60} \mathrm{Fe}$ are shown for different models, together with the statistical variance. Shown are the $1 \sigma$ and $2 \sigma$ statistical deviations (the intervals containing $68 \%$ and $95 \%$ of the Monte Carlo simulations) for a population of 100 stars in the $8-120 M_{\odot}$ range $^{2}$, corresponding to a typical nearby star forming region (for example the number of massive stars formed within the last $15 \mathrm{Myr}$ in the Orion OB1 association is estimated to be close to 100 Brown et al. 1994). We note that the $8-120 M_{\odot}$ range includes more massive stars than are observed in many nearby regions. However, when a probabilistic description (such as our Monte Carlo simulations) is assumed, the limit should be the most massive star theoretically possible in the cluster. Observed clusters correspond to random realizations of the IMF and the most massive stars in these can therefore have much lower masses than the upper limit of $120 M_{\odot}$ (see also Sect. 4 where the contributions of various initial mass ranges to the observables are shown).

21 star in this range corresponds to 381,140 and 188 stars in the 0.1-120 $M_{\odot}$ range for the Salpeter (Salpeter 1955), Kroupa (Kroupa 2001) and the Scalo (Scalo 1986) mass functions, respectively, and to $13 \%, 18 \%$ and $5 \%$ of the stellar mass.

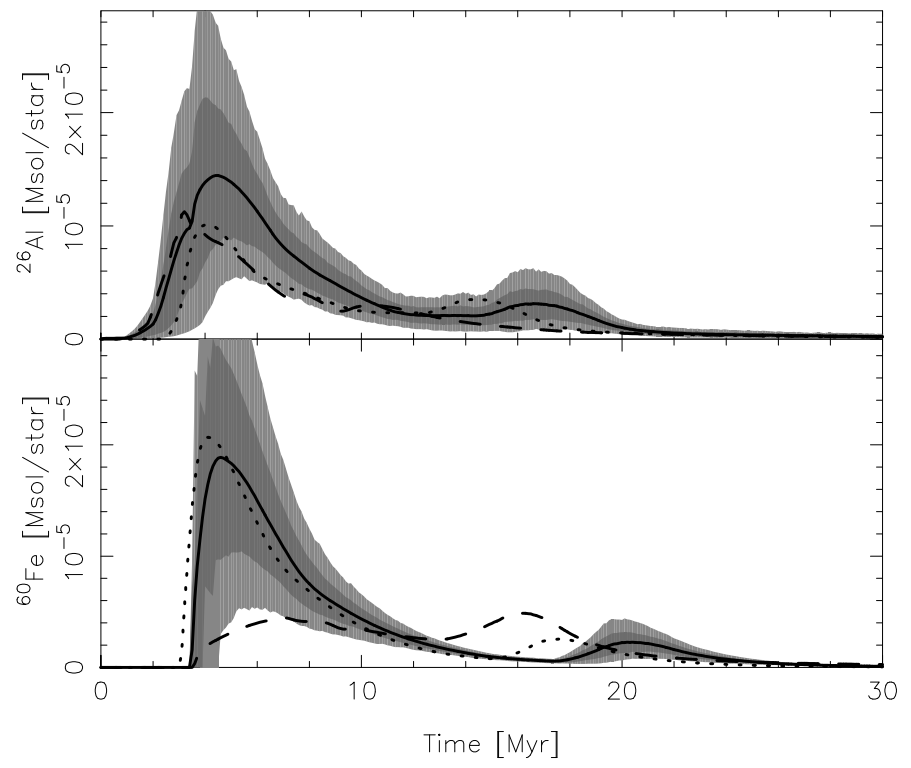

Fig. 3. Time profiles of ${ }^{26} \mathrm{Al}$ (top) and ${ }^{60} \mathrm{Fe}$ (bottom) for a coeval population of stars. The solid lines indicate the average profiles for the geneva05 stellar models with the yieldsLC2006 supernova yields. The the dark and light grey regions show the $1 \sigma$ and the $2 \sigma$ deviations for a population of 100 stars between 8 and $120 M_{\odot}$, based on Monte Carlo simulations. The dashed and dotted lines show our main alternative models. Dashed: geneva97 stellar tracks with yieldsWW95 supernova yields. Dotted: LCO6 stellar tracks with yieldsLC2006 supernova yields.

From Fig. 3 it is obvious that for relatively small populations, it is essential that these statistical effects are taken into account, when interpreting observations. Also very interesting is the ratio between the observable strengths of the ${ }^{60} \mathrm{Fe}$ and ${ }^{26} \mathrm{Al} \gamma$-ray lines. This is shown in Fig. 4 for the same models as in Fig. 3. For much of the time, this ratio places stronger constraints on the stellar models than the individual observations of ${ }^{26} \mathrm{Al}$ and ${ }^{60} \mathrm{Fe}$. This is due to the fact that the emissions of these two elements are correlated. The strong increase in the ${ }^{60} \mathrm{Fe} /{ }^{26} \mathrm{Al}$ ratio seen around the lifetime of an $8 M_{\odot}$ star ( 35-50 Myr, depending on the stellar model) is simply an effect of the longer lifetime of ${ }^{60} \mathrm{Fe}$, when the elements are not being replenished (non-steady state situation).

Recent results (Rugel, in preparation) indicate that the lifetime of ${ }^{60} \mathrm{Fe}$ is significantly $(\sim 3.8 \mathrm{Myr})$ longer than the commonly used lifetime of $\sim 2$ Myr. It is unclear if a different lifetime would have any significant impact on the nucleosynthesis of ${ }^{60} \mathrm{Fe}$, and a study of this is beyond the scope of this paper. In Fig. 5 we show the effect on the time profile of ${ }^{60} \mathrm{Fe}$ for our default model, assuming that the amount of ${ }^{60} \mathrm{Fe}$ released in the supernova explosions is unchanged. Due to the longer decay timescale, the build-up of the isotope is larger, and in the period after the main peak (5-15 Myr after the star formation), the amount of ${ }^{60} \mathrm{Fe}$ present in the ISM is approximately twice as large as for the shorter ${ }^{60} \mathrm{Fe}$ lifetime. Note that the effect on the observed flux is different. While there is more ${ }^{60} \mathrm{Fe}$ present in the ISM, the $\gamma$-ray emission per unit mass is decreased due to the longer decay timescale. This is illustrated by the grey dashed line in Fig. 5: the integrated amount of $\gamma$-ray emission is unchanged (since the amount of ${ }^{60} \mathrm{Fe}$ released from the stars is unchanged), but the distribution is slightly shifted to later times. The only significant effect is the lower peak at $\sim 5 \mathrm{Myr}$. It should be noted that in a constant star-formation scenario (steady-state), 


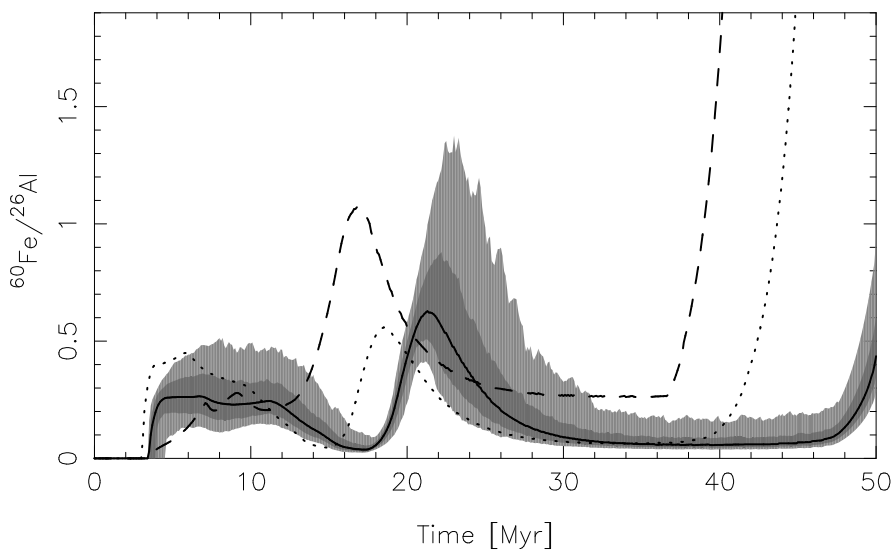

Fig. 4. Time profiles of ${ }^{60} \mathrm{Fe} /{ }^{26} \mathrm{Al}$ emission ratio for a coeval population of stars. Legends same as Fig. 3.

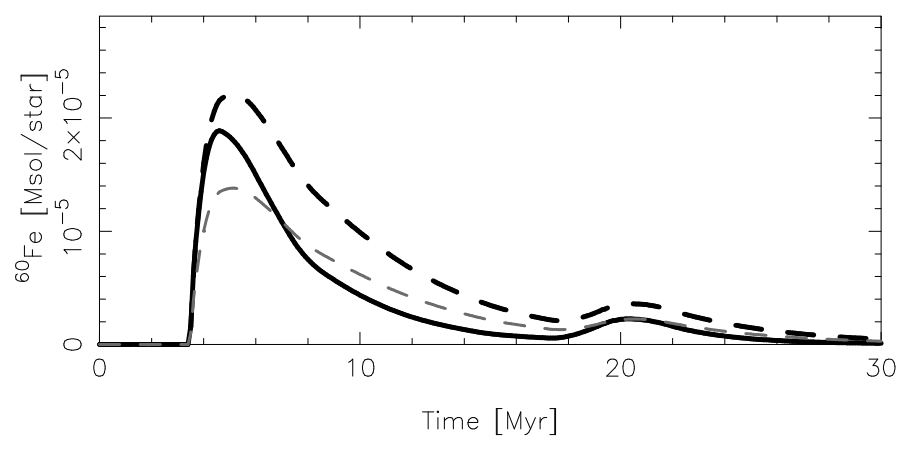

Fig. 5. Time profiles of ${ }^{60} \mathrm{Fe}$ for a coeval population of stars for the two different decay timescales. The solid line is the same as in Fig. 3, with an average lifetime of $\sim 2 \mathrm{Myr}$, whereas the black dashed line is for an average lifetime of $\sim 3.8 \mathrm{Myr}$. The grey dashed line indicates the observational difference (the amount of ${ }^{60} \mathrm{Fe}$ that would be inferred from observations if a lifetime of $2 \mathrm{Myr}$ is assumed, in the case the lifetime actually is $3.8 \mathrm{Myr}$.

the measured ratio of ${ }^{60} \mathrm{Fe} /{ }^{26} \mathrm{Al}$ emission is independent of the decay times of the two isotopes (although the ratio of ${ }^{60} \mathrm{Fe} /{ }^{26} \mathrm{Al}$ does depend on the decay times).

\subsection{Energy and mass}

The energy and mass ejection rates are the most important parameters for the evolution of the interstellar medium in the vicinity of young stars. Winds and SN ejecta compress the surrounding medium, creating wind-driven bubbles, supernova remnants, and even super-bubbles in the case of multiplets of massive stars. These stellar systems dissociate the surrounding molecular clouds and may induce star-formation within these. In Fig. 6, the "kinetic luminosities" of the winds and the supernova explosions are shown for the three sets of stellar tracks. In the first 3-4 Myr, no stars explode, and the kinetic luminosity originates exclusively from stellar winds. The mechanicial power of these sustained winds is roughly equal to the average power of punctuated supernova explosions, once these set in. It is important to note that integrated over the first $10 \mathrm{Myr}$, the total energy emitted in winds is actually larger than the energy associated with the supernova explosions. This is important for shaping the medium around a star-forming region. There are large differences between the various stellar models. In particular it is worth noting that while decreased mass-loss rates in rotating stellar models obviously decrease the wind power, this is compensated for by

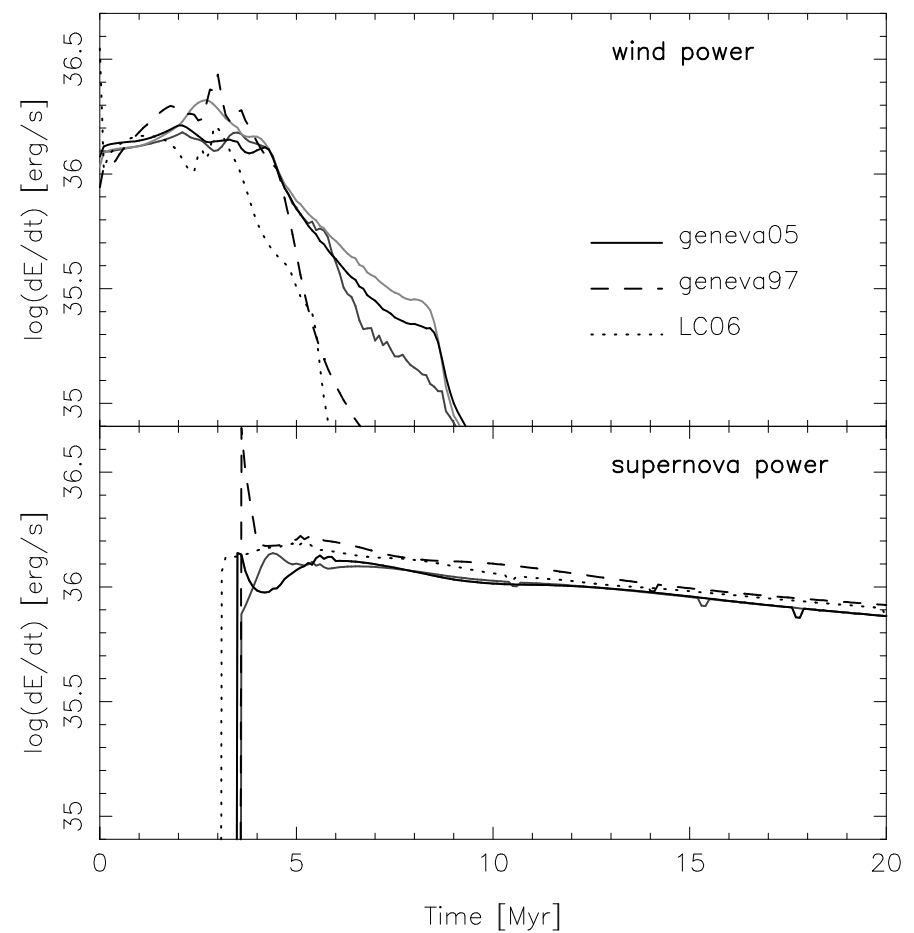

Fig. 6. Time profiles of the wind (top) and supernova power per star from a coeval population of stars in the $8-120 M_{\odot}$ mass range, using the various stellar models with the wind08 wind velocities. The solid dark grey line is associated with the geneva05alt evolutionary tracks, and the solid light grey line corresponds to the geneva05 stellar models with wind00.

their longer lifetimes. Furthermore the longer stellar lifetimes lower the supernova rate (except at late times $\gtrsim 35 \mathrm{Myr}$, where the supernova rate for non-rotating stars becomes zero, whereas the rotating stars still explode until $\sim 45 \mathrm{Myr}$ ), and therefore the early dominance of wind energy is even stronger.

A comparison between the two wind prescriptions wind 00 and wind08 shows significant differences. Wind velocities of different types of massive stars still have very large uncertainties, and this translates into large uncertainties in the implicated wind power. As the most massive stars explode after $~ 5 \mathrm{Myr}$, the wind power decreases rapidly, thereafter the supernova rate decreases steadily until stars with masses $\sim 8 M_{\odot}$ explode, and the energy deposition in the ISM becomes negligible.

In Fig. 7 the average total kinetic luminosity and the mass ejection rate are compared through Monte Carlo simulations of a small population of stars. For the Monte Carlo simulations the power was averaged over $1 \mathrm{Myr}$, and still the variations are very large, especially in the later stages, where only few supernovae are likely to explode within a time interval. When studying small regions it is important to note these statistical effects, especially for processes with shorter timescales, where the variability of a local supernova rate increases drastically. For example the $1 \sigma$ range of the supernova rate around $10 \mathrm{Myr}$ covers more than a factor of 100 when averaged over a timescale of $0.1 \mathrm{Myr}$, similar to the replenishment timescale of the X-ray emitting gas in the region of Orion studied by Güdel et al. (2008). We note that the variation in the time integrated energy (studied by Cerviño et al. 2001, 2002), important for studying the X-ray bubble expansion at larger scales, is smaller, since it only depends on the number of supernova explosions and not on the specific explosion times. The mass ejection rate shown in the lower panel of Fig. 7 is more strongly dominated by the ejection through 


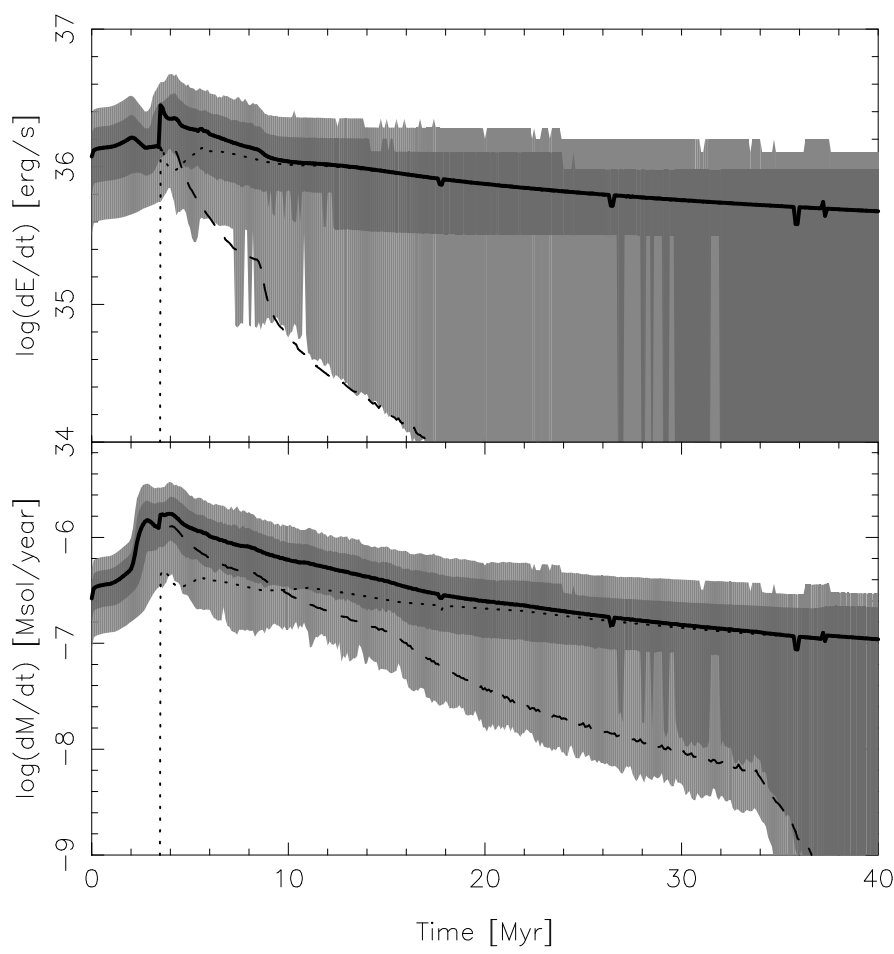

Fig. 7. Time profiles energy (top) and mass (bottom) ejection rates, with $1 \sigma$ (dark grey) and $2 \sigma$ (light grey) Monte Carlo deviations for a population of 100 stars between 8 and $120 M_{\odot}$. The solid line indicates the full outputs, with the dashed and dotted lines indicating the contributions from stellar winds and supernova explosions, respectively. The Monte Carlo outputs were averaged over $1 \mathrm{Myr}$.

stellar winds, and as this is a continuous process, the variations are relatively smaller.

\subsection{UV radiation}

The bulk of the ionizing flux is provided by hot stars that disappear after only a few Myr. This is clearly seen in Fig. 8, where the time profile of the ionizing flux is shown. The flux is high and roughly constant during the first $2 \mathrm{Myr}$, after which it declines. After less than $10 \mathrm{Myr}$ it has declined by a factor of more than 100 , and the rate of decline steepens further. There are still large uncertainties in the modelling of non-LTE expanding atmospheres. Comparing the results with a now superseded set of stellar atmospheres shows only minor differences, but the comparison with the non-rotating L\&C stellar models shows dramatic differences. So while the correct atmosphere modelling is important for the study of single stars and for understanding the UV spectrum, the stellar evolution modelling is much more important for the understanding of the interactions between groups of massive stars and their surroundings. It is important to note that for small stellar associations, the statistical deviations from the mean are very large (see e.g. Fig. 4 in Cerviño et al. 2000b, for the deviation for associations of different masses).

\subsection{Statistical properties of the stellar populations}

Above we described the average outputs of the ${ }^{26} \mathrm{Al},{ }^{60} \mathrm{Fe}$, energy, mass and UV radiation from young stellar populations, together with examples of their variability, based on Monte Carlo simulations. For a better understanding of the statistical properties of the stellar distributions, we also made use of the statistical

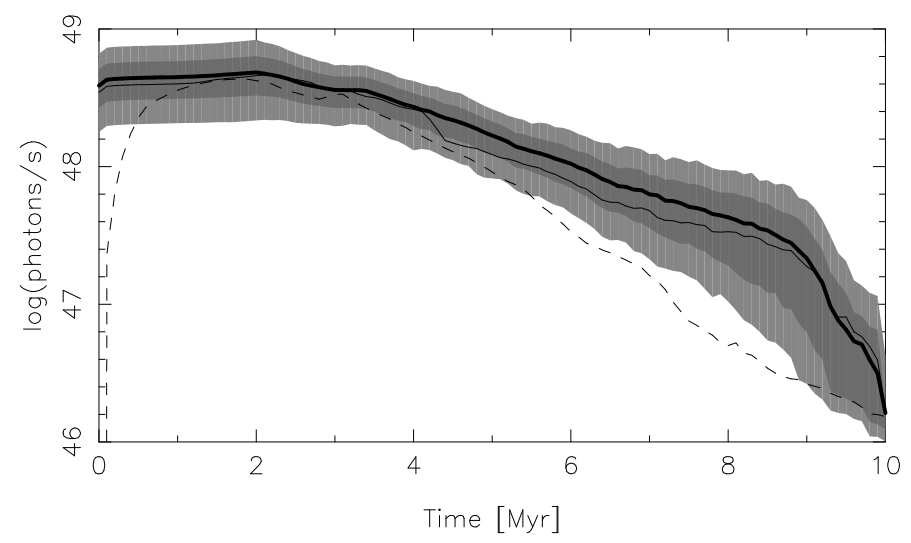

Fig. 8. Time profiles of the ionizing flux per star, with $1 \sigma$ (dark grey) and $2 \sigma$ (light grey) Monte Carlo deviations for a population of 100 stars between 8 and $120 M_{\odot}$, using the geneva05 stellar models with the atmosMS atmosphere models. The thin solid line was produced by using the atmosOLD atmosphere models, whereas the dashed line uses the LCO6 stellar models and the atmosMS atmospheres.

methods of Cerviño \& Luridiana (2006). At a given time, these methods allow the analytical approximation of the probability distribution of a quantity. We calculate the raw moments of the distribution

$\mu_{n}=\int_{0}^{\infty}(l-a)^{n} \phi(l) \mathrm{d} l$,

where $n$ is the integer order of the moment, $l$ is the physical quantity for which we investigate the distribution, and $\phi(l)$ is the probability for a specific value of $l$. The parameter $a$ is the average value of $l$ :

$a=\int_{0}^{\infty} l \phi(l) \mathrm{d} l$

From these we find the skewness

$\gamma_{1}=\frac{\mu_{3}}{\mu_{2}^{3 / 2}}$

and the kurtosis

$\gamma_{2}=\frac{\mu_{4}}{\mu_{2}^{2}}-3$

of the distribution. For an ensemble of $N$ stars, the distribution expressed in normal form (i.e., transformed to a distribution with zero mean and unit variance) has a skewness $\Gamma_{1}=\gamma_{1} / \sqrt{N}$ and kurtosis $\Gamma_{2}=\gamma_{2} / N$. With these we can estimate the distribution using a Gaussian multiplied by Edgeworth's series (see e.g. Blinnikov \& Moessner 1998; Cerviño \& Luridiana 2006), which, truncated to 2 terms, is:

$$
\begin{aligned}
\varphi_{\mathrm{L}_{\mathrm{tot}}}(x)= & \frac{1}{\sqrt{2 \pi}} \mathrm{e}^{-\frac{1}{2} x^{2}} \\
& \times\left(1+\frac{1}{6} \Gamma_{1}\left(x^{3}-3 x\right)+\frac{1}{24} \Gamma_{2}\left(x^{4}-6 x^{2}+3\right)\right. \\
& \left.+\frac{1}{72} \Gamma_{1}^{2}\left(x^{6}-15 x^{4}+45 x^{2}-15\right)\right)
\end{aligned}
$$

For very small populations the discrepancy between the Edgeworth approximation and the real distribution becomes large. Cerviño \& Luridiana (2006) therefore derived analytical estimates of the necessary population size to allow the use 


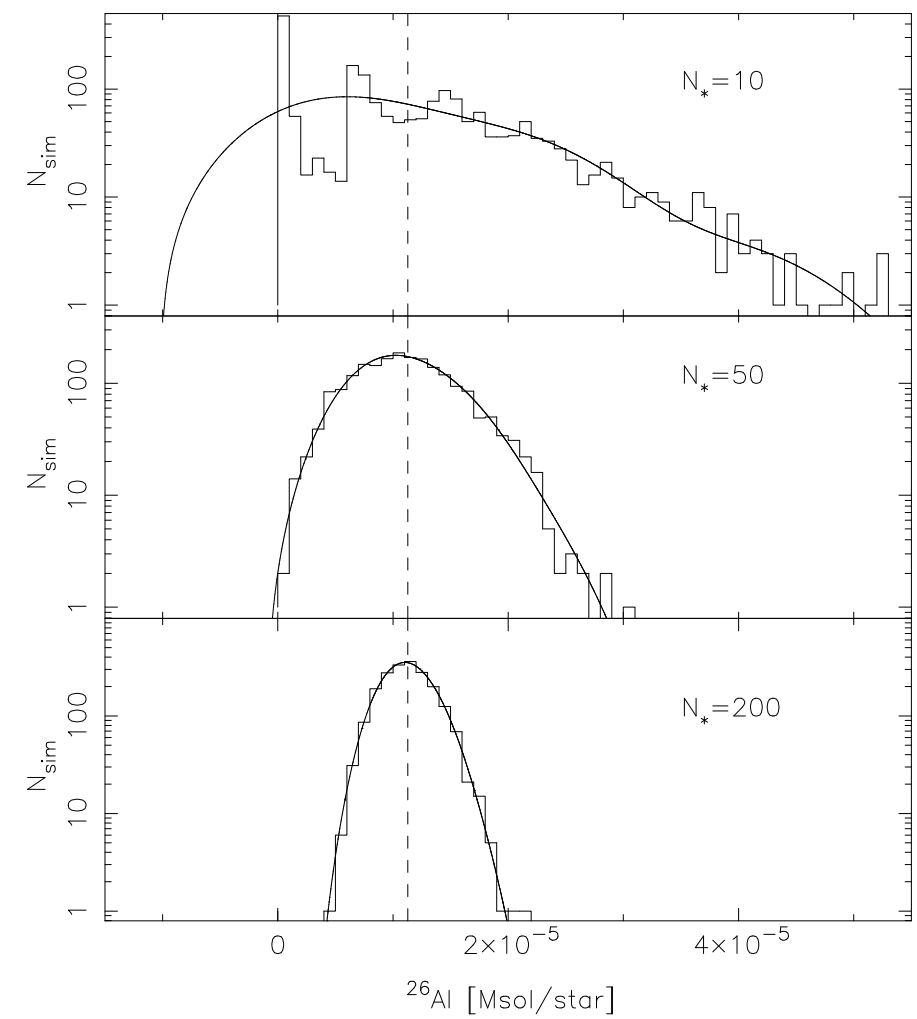

Fig. 9. The statistical distribution of ${ }^{26} \mathrm{Al}$ in the interstellar medium around a cluster of 10,50 and 200 massive stars $\left(N_{*}\right)$ in the 8-120 $M_{\odot}$ range in the top, middle and bottom panels, respectively. The distribution is given for an age of $6 \mathrm{Myr}$. The histograms show the distribution of 2000 Monte Carlo simulations, while the solid lines are based on the Edgeworth approximation. The vertical dashed line is the average value. geneva05 stellar models and LC2006 supernova yields were assumed.

of their approximation, and also when it is possible to use a Gaussian approximation. In Fig. 9 an example of the probability distribution for ${ }^{26} \mathrm{Al}$ is shown, and the Edgeworth approximation is compared to Monte Carlo simulations. It can be seen that while for very low numbers of stars, the distribution can only be investigated using Monte Carlo simulations, whereas for intermediate cases Edgeworth's approximation is useful. Figure 10 shows the skewness and the kurtosis of the distribution. In Fig. 11 the minimum number of stars needed for an adequate approximation (within $10 \%$ ) in the $\pm 3 \sigma$ interval is shown for Egdeworths approximation and for the Gaussian approximation. Evidently star-forming regions need to have a very large number of massive stars (in the best case 1000 stars above $8 M_{\odot}$ ) for Gaussian statistics to be applicable. But even outside the Gaussian regime, the evolution of $\gamma_{1}$ and $\gamma_{2}$ values allow us to obtain some inferences about the results of the Monte Carlo simulations with low numbers of stars without performing them. First of all, $\gamma_{1}$ is a measure of the asymmetry of the distribution. A positive $\gamma_{1}$ means a "L-shape" distribution. A large $\gamma_{2}$ means that the distribution is both more peaked than Gaussian distributions near the maximum, and more flat than Gaussian in the tails. Large $\gamma_{1}$ and $\gamma_{2}$ values together reflects then (a) the most probable value is peaked in a value different from the mean and; (b) there is a non-negligible high luminosity tail in the distribution.

Appling these concepts to our study, the large values in $\gamma_{1}$ and $\gamma_{2}$ at young ages (before the onset of the $\mathrm{SN}$ phase in ${ }^{26} \mathrm{Al}$ production) shows the large impact of particular high massive stars in the the integrated production of ${ }^{26} \mathrm{Al}$. It implies that, for low mass clusters, the current ${ }^{26} \mathrm{Al}$ produced by an individual

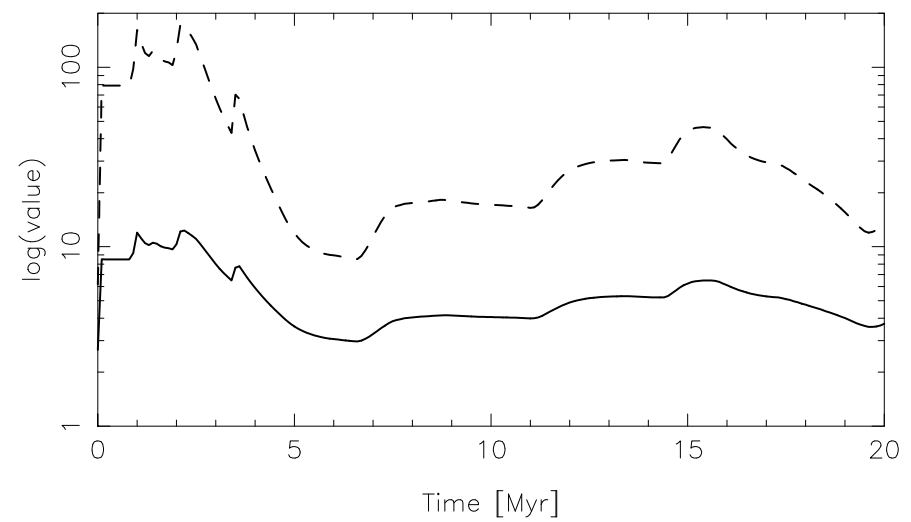

Fig. 10. The skewness, $\gamma_{1}$ and the kurtosis, $\gamma_{2}$ of the ${ }^{26} \mathrm{Al}$ distribution for a population of stars in the $8-120 M_{\odot}$ mass range.

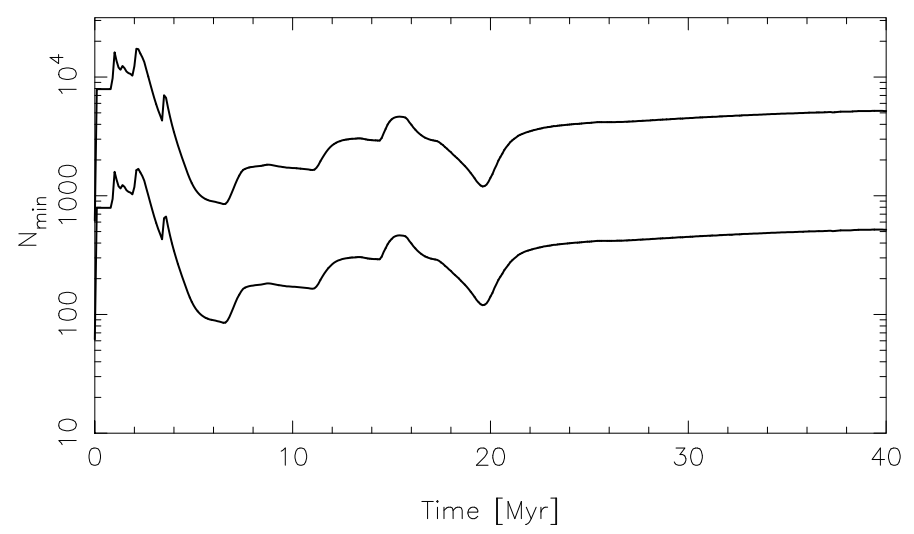

Fig. 11. Lower limits on the number of stars in the $8-120 M_{\odot}$ range needed for the statistical distribution of ${ }^{26} \mathrm{Al}$ to be approximated by a Gaussian (upper line) and by Edgeworth's approximation (lower line). geneva05 stellar models and LC2006 supernova yields were assumed.

cluster is strongly dependent on the most massive star the cluster has formed, so (provided the cluster age is known from an independent method) it would be possible to estimate the value of this most massive star. Of course, since we are only able to obtain statistical inferences in the low cluster mass regime, we need to take into account the maximum possible $M_{\mathrm{up}}$ value, which naturally include the case where such more massive stars are not present in the cluster due to the particular realization of the IMF performed by Nature in a given cluster. These effects are clearly visible even for cluster with 10 massive stars in the top pannel of Fig. 9. In the pure SN dominated phase (ages larger than $20 \mathrm{Myr}$ ), $\gamma_{1}$ and $\gamma_{2}$ are almost constant, without significant structure and are both slowly increasing with age (cf. Fig. 11). A simple consequence of the slow decay of the SN rate at these ages is, (a) an increase of sampling effects due to the declining number of possible SNe within a given time window; and (b) a decrease in the sensitivity to which star has exploded as a SN in the ${ }^{26} \mathrm{Al}$ ejection, as there is not much variation in the ${ }^{26} \mathrm{Al}$ ejection from $\mathrm{SNe}$ at these late times. The intermediate age range between 7 an 20 Myr shows how the stars that explode in this age range have different amounts of ${ }^{26} \mathrm{Al}$ ejections.

Finally, in Fig. 12 the Pearson correlation coefficient between the $\gamma$-ray emission from nucleosynthesis products and the hydrogen ionizing radiation is shown. The use of these correlation coefficients allows us to establish the probability of obtaining the value of one of the observables, given that the value of the other observable is known. A correlation coefficient equal 


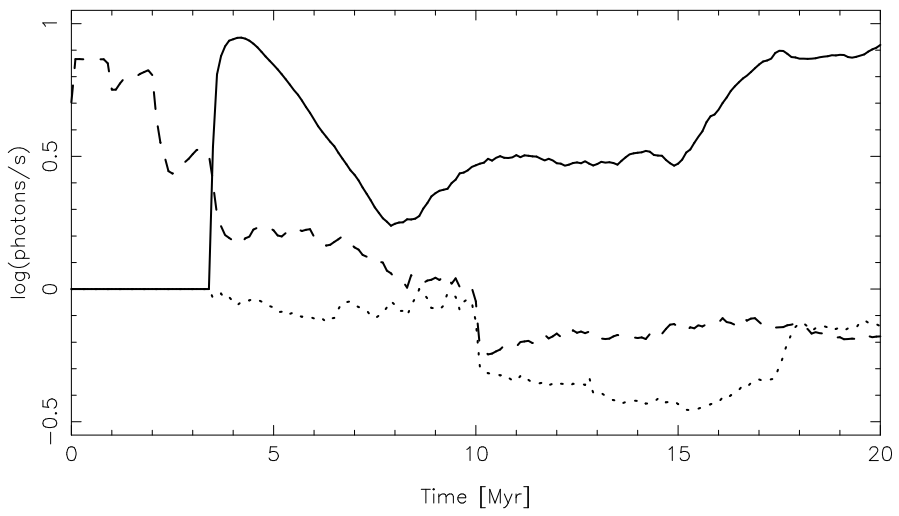

Fig. 12. The Pearson correlation coefficient between the ${ }^{26} \mathrm{Al}$ and ${ }^{60} \mathrm{Fe}$ $\gamma$-ray emission (solid line), the ${ }^{26} \mathrm{Al} \gamma$-ray and the UV emission (dashed line) and the ${ }^{60} \mathrm{Fe} \gamma$-ray and the UV emission (dotted line), as a function of time for our default model.

to zero implies that the two associated quantities do not contain information about each other. There is no strong correlation between the $\gamma$-rays from ${ }^{60} \mathrm{Fe}$ and UV radiation, and therefore the overall UV radiation does not know anything about the ${ }^{60} \mathrm{Fe}$ production. The strong correlation between the ${ }^{26} \mathrm{Al} \gamma$-ray emission (from the ${ }^{26} \mathrm{Al}$ ejected in the stellar winds) and the UV radiation at young ages refects that both components are produced by related sources: note that there is a time delay between the UV and the ${ }^{26} \mathrm{Al}$ emission, so the sources that produce both quantities are not the same. The ${ }^{26} \mathrm{Al}-\mathrm{UV}$ correlation decreases with time, since the stars that produce the UV emission are unrelated to the stars that explode as $\mathrm{SN}$ and produce most of the ${ }^{26} \mathrm{Al}$ at ages later than $6 \mathrm{Myr}$. Finally, the $\gamma$-rays from ${ }^{60} \mathrm{Fe}$ and ${ }^{26} \mathrm{Al}$ are highly correlated but the correlation does not reach a value of 1 , reflecting the fact that the overall production comes from similar, but not the same, stellar sub-populations.

\section{Discussion}

The outflow (energy and matter) from young stars into the ISM determines the interplay between star formation and galaxy evolution. Our population synthesis code predicts important stellar outputs necessary to study these processes in more detail. The emission of radioactive isotopes is important for such studies, as they are a unique way of tracing the output from the massive stars directly, globally (Milky Way wide) as well as the spatial distribution of the ejecta around young stellar clusters. Many aspects of stellar evolution of massive stars are quite uncertain, and often the effects of these on the properties of a stellar cluster are not directly apparent. Population synthesis studies allow to test the impact of changes in specific ingredients and processes of stellar evolution models.

Our study of the ejection of the radioactive elements ${ }^{26} \mathrm{Al}$ and ${ }^{60} \mathrm{Fe}$ shows considerable theoretical uncertainties on the ejection rates and time profiles. Especially the amount of ${ }^{60} \mathrm{Fe}$ ejected from the most massive stars is sensitive to the structure of the stars in the final evolutionary stages. The emission of ${ }^{26} \mathrm{Al}$ varies by less than a factor $\sim 2$, and the ratio of these two elements is therefore an important diagnostic for the late stages of stellar evolution of very massive stars. An important ingredient is mass loss from the massive stars. Despite recently improved understanding of this process, the rate of mass loss is still subject of debate, with proposed downward changes of up to an order of magnitude. One effect of lower mass loss rates is an increase of the stellar core sizes at late burning stages.

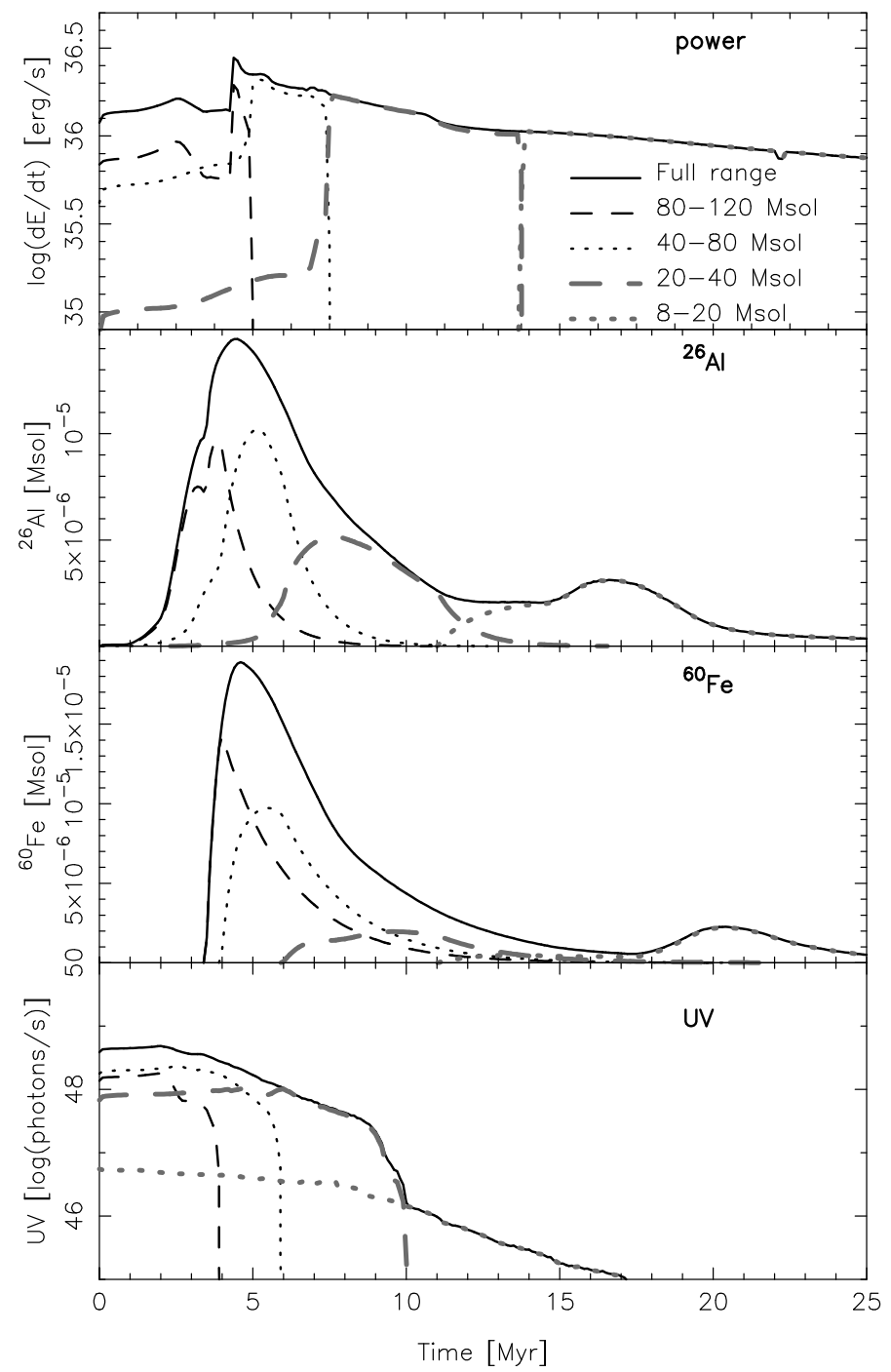

Fig. 13. The time profiles of the kinetic power, ${ }^{26} \mathrm{Al},{ }^{60} \mathrm{Fe}$ and UV, with the contributions from 4 mass ranges. The solid lines are for the full range of masses (same as the solid lines in Figs. 3 and 7.

The ${ }^{60} \mathrm{Fe}$ production is correspondingly enhanced, and the wind ejection of ${ }^{26} \mathrm{Al}$ is decreased. For non-rotating stellar models, with the currently preferred mass-loss rates (Limongi \& Chieffi 2006) the Galactic ${ }^{60} \mathrm{Fe} /{ }^{26} \mathrm{Al}$ ratio is overpredicted with respect to measurements (Wang et al. 2007), leading Limongi \& Chieffi (2006) to suggest changes in the IMF or a relatively low upper integration limit. For lower mass-loss rates this problem would be enhanced and this is a strong argument against very low massloss rates for non-rotating stellar tracks (although it is important to note that the uncertainties in the nucleosynthesis of ${ }^{60} \mathrm{Fe}$ and ${ }^{26} \mathrm{Al}$ are too large for the argument to be definitive at this time). Stellar rotation can strongly enhance the mass lost from stars, mainly from increasing the duration of the WR-phases. However, rotation also tends to increase the size of the stellar core, and without calculations of the later evolutionary stages of such stars, it is difficult to predict the explosively ejected nucleosynthetic yields. The observed average Galactic ${ }^{60} \mathrm{Fe} /{ }^{26} \mathrm{Al}$ ratio provides interesting constraints on the models, but observations of specific regions are also important, as the time profile and the statistical variability can improve our understanding of massive star evolution considerably. While the study of the ratio in individual star-forming regions will not be possible for 
many years, it is expected that it will at least be possible to divide the Milky Way into a few sections for this purpose at the end of the INTEGRAL mission. The main problem is the detection limit of the ${ }^{60} \mathrm{Fe} \gamma$-rays. On the other hand, the prospects for studying the time profile of ${ }^{26} \mathrm{Al}$ are good, and in subsequent papers we will apply our population synthesis tool to the Galactic ${ }^{26} \mathrm{Al}$ distribution, and to individual star forming regions, such as Orion. Figure 13 shows that at different intervals, the output of energy and matter from the stars is dominated by different ranges of initial stellar masses. Therefore the analysis of different regions with non-steady star formation can be used to explore the physics of stars in quite narrow mass ranges.

${ }^{26} \mathrm{Al}$ and ${ }^{60} \mathrm{Fe}$, together with many additional isotopes, are emitted into the ISM with very high velocities. These ejecta will move away from the star clusters, which has been observed in the Orion region where a part of the ${ }^{26} \mathrm{Al}$ signal is off-set from the star clusters, and coincides with the Eridanus bubble formed by the outflowing hot gas (Diehl 2002). The spread of ${ }^{26} \mathrm{Al}$ can therefore trace the mixing of young stellar ejecta into the ISM, a process that is relatively poorly understood but important for proper modelling of the evolution of galaxies. Our code predicts the mass, energy and ionizing radiation output of the stellar clusters, which are the most important parameters for the interaction with the ISM. Our results show that stellar winds are important for the turbulent state of the ISM. In the first $\sim 6 \mathrm{Myr}$ the power from the winds clearly dominates, being as strong as the subsequent power injected by supernovae, even if the total energy of the supernova explosions is higher than the total wind energy, as they explode over a much longer timescale. For the winds the most massive stars are very important, even if there are relatively few of them, as they lose most of their mass through winds with very high velocities in the WR-phases. Estimating the wind power from a typical O-type star and multiplying by the number of stars in a cluster will underestimate the wind power by more than an order of magnitude. When comparing the mechanically derived power from different stellar models, we find that, while the mass-loss rates are smaller by a factor $\sim 2-3$ in the geneva05 models compared to the geneva97 models, the wind power is actually more dominates with the geneva 05 models. On the other hand, for the LCO6 models that also have the lower mass-loss rates, the role of the winds is somewhat diminished. The reason for the enhanced wind importance when using the rotating geneva 05 models is caused by several factors, the most important ones being the greatly increased time spent in WR-phases where the mass-loss rate and wind velocity is very high, and the longer stellar lifetimes causing a decrease in the supernova rate. We note that in all models the wind power is more important than commonly recognized (e.g. in the study of supernova driven bubbles). This means that for stellar clusters, the supernova shells expand into a pre-existing cavity, rather than in a dense star-forming ISM. For example Cho \& Kang (2008) find that the volume of a supernova bubble is $\sim 2-3$ times larger and also hotter when the winds are taken into account, even though the wind power assumed in their study is very low compared to our estimates. The flow of wind and supernova ejecta inside cavities can be very complex (MacLow et al. 2005), and the propagation is likely dominated by turbulent diffusion from magnetic field irregularities caused by the stellar winds and supernovae (Balsara \& Kim 2005; Parizot 2004). Part of the gas will be thermalized near the stellar association due to wind-wind collisions or by a termination shock against the turbulent medium inside the cavity, and this can be observed as a hot X-ray emitting plasma (Townsley et al. 2003; Güdel et al. 2008). However the majority of the mass will expand into the low density cavity.
The effective propagation velocity is very uncertain, and is expected to be in the range $100-1000 \mathrm{~km} \mathrm{~s}^{-1}$ (for example Balsara et al. 2008, finds velocities of $\sim 200 \mathrm{~km} \mathrm{~s}^{-1}$ ). ${ }^{26} \mathrm{Al}$ and ${ }^{60} \mathrm{Fe}$ can be important for measuring this velocity, as their lifetimes are similar to the time it takes for the ejecta to cross a cavity blown by a young stellar cluster. If the velocity is high, these elements will quickly reach the wall of the cavity, and the brightness distribution will be given by the geometry of the walls. For slower propagation the fresh ejecta will be distributed inside the cavity, and the measured line widths will reflect the turbulence inside the cavity directly.

\section{Conclusions}

We have developed a populations synthesis code for the study of Galactic OB associations. The main aim of this work is the prediction of the output of energy, gas, ${ }^{26} \mathrm{Al},{ }^{60} \mathrm{Fe}$ and ionizing photons of a population of massive stars. We describe the dependence on the input physics, especially rotating stellar models (Meynet \& Maeder 2005; Palacios et al. 2005). We show that energy and matter output depend on the choice of stellar evolutionary and supernova models, whereas other parameters, such as the speeds of the winds and the stellar atmospheres only play secondary roles. This is a problem for the correct modelling of star-forming regions, and also means that comparison with observations (for example the $\gamma$-ray emission from radioactive isotopes) can potentially yield important constraints for stellar and supernova models. Also the statistical variations for small numbers of stars in individual regions are addressed, which is important when interpreting observations of nearby, small starforming regions.

Our study shows that the ejected ${ }^{26} \mathrm{Al}$ and wind power from a population of massive stars is strongly enhanced by the effects of stellar rotation. Despite the recent downward revision of the mass-loss rates from massive stars by a factor of $2-3$, the amount of ${ }^{26} \mathrm{Al}$ and the importance of the wind power is actually increased by including stellar rotation.

Acknowledgements. This research was supported by the DFG cluster of excellence "Origin and Structure of the Universe" (http://www. universe-cluster.de). M.C. is supported by the Spanish PNAyA project through FEDER funding of project AYA207-64712.

\section{References}

Balsara, D. S., \& Kim, J. 2005, ApJ, 634, 390

Balsara, D. S., Bendinelli, A. J., Tilley, D. A., Massari, A. R., \& Howk, J. C. 2008, MNRAS, 386, 642

Barlow, M. J., Smith, L. J., \& Willis, A. J. 1981, MNRAS, 196, 101

Blinnikov, S., \& Moessner, R. 1998, A\&AS, 130, 193

Brown, A. G. A., de Geus, E. J., \& de Zeeuw, P. T. 1994, A\&A, 289, 101

Bruzual, A. G. 1983, ApJ, 273, 105

Bruzual, G., \& Charlot, S. 2003, MNRAS, 344, 1000

Castor, J. I., Abbott, D. C., \& Klein, R. I. 1975, 195, 157

Cerviño, M., \& Luridiana, V. 2006, A\&A, 451, 475

Cerviño, M., Knödlseder, J., Schaerer, D., von Ballmoos, P., \& Meynet, G. 2000a, A\&A, 363, 970

Cerviño, M., Luridiana, V., \& Castander, F. J. 2000b, A\&A, 360, 5

Cerviño, M., Gómez-Flechoso, M. A., Castander, F. J., et al. 2001, A\&A, 376, 422

Cerviño, M., Mas-Hesse, J. M., \& Kunth, D. 2002, A\&A, 392, 19

Cho, H., \& Kang, H. 2008, New Astron., 13, 163

Diehl, R. 2002, New Astron. Rev., 46, 547

Diehl, R., Dupraz, C., Bennet, K., et al. 1995, A\&A, 298, 445

Diehl, R., Halloin, H., Kretschmer, K., et al. 2006, Nature, 439, 45

Eldridge, J. J., \& Vink, J. S. 2006, A\&A, 452, 295

Eldridge, J. J., Izzard, R. G., \& Tout, C. A. 2008, MNRAS, 384

Güdel, M., Briggs, K. R., Montmerle, T., et al. 2008, Science, 319, 309

Hamann, W.-R., Gräfener, G., \& Liermann, A. 2006, A\&A, 457, 1015

Harris, M. J., Knödlseder, J., Jean, P., et al. 2005, A\&A, 433, 49 
Howarth, I. D., \& Prinja, R. K. 1989, ApJS, 69, 527

van der Hucht, K. A., Williams, P. M., \& The, P. S. 1987, QJRAS, 28, 254

Hunter, I., Dufton, P. L., Smartt, S. J., et al. 2007, A\&A, 466, 277

de Jager, C., Nieuwenhuijzen, H., \& van der Hucht, K. A. 1988, A\&AS, 72, 259

Jones, T. W., Rudnick, L., Jun, B.-I., et al. 1998, PASP, 110, 125

Knödlseder, J., Bennet, K., Bloemen, H., et al. 1999, A\&A, 344, 68

Kroupa, P. 2001, MNRAS, 322, 231

Kurucz, R. L. 1992, in The Stellar Populations of Galaxies, IAU Symp., 149, 225

Lamers, H. J. G. L. M., \& Morton, D. C. 1976, ApJS, 32, 715

Lamers, H. J. G. L. M., Snow, T. P., \& Lindholm, D. M. 1995, ApJ, 455, 269

Langer, N., Braun, H., \& Wellenstein, S. 1998, in Proceedings of the 9th Workshop on Nuclear Astrophysics, ed. W. Hillebrandt, \& E. Müller, 18

Leitherer, C., Robert, C., \& Drissen, L. 1992, ApJ, 401, 596

Leitherer, C., Schaerer, D., Goldader, J. D., et al. 1999, ApJS, 123, 3

Limongi, M., \& Chieffi, A. 2006, ApJ, 647, 483

Lozinskaya, T. A. 1992, Supernovae and stellar wind in the interstellar medium (New York, USA: AIP)

MacLow, M., Balsara, K., Kim, J., \& de Avillez, M. A. 2005, ApJ, 626, 864

Maeder, A. 1990, A\&AS, 84, 139

Maeder, A., \& Conti, P. S. 1994, ARA\&A, 32, 227

Maeder, A., \& Meynet, G. 1994, A\&A, 287, 803

Maeder, A., Georgy, C., \& Meynet, G. 2008, A\&A, 479, L37

Martins, F., Schaerer, D., \& Hillier, D. J. 2005, A\&A, 436, 1049

Meynet, G., \& Maeder, A. 2005, A\&A, 429, 581

Meynet, G., Gould, M., Prantzos, N., et al. 1997, A\&A, 320, 460

Niedzielski, A., Skorzynski, W. 2002, Acta Astron., 52, 81

Nugis, T., \& Lamers, H. J. G. L. M. 2000, A\&A, 360, 227

Palacios, A., Meynet, G., Vuissoz, C., et al. 2005, A\&A, 429, 613
Panagia, N. 1973, AJ, 78, 929

Parizot, E., Marcowith, A., van der Swaluw, E., Bykov, A. M., \& Tatischeff, V. 2004, A\&A, 424, 747

Plüschke, S. 2001, 26Al Ursprung und OB Associationen, Ph.D. Thesis, Technische Universität München, Germany

Prantzos, N., \& Diehl, R. 1996, Phys. Rep., 267, 1

Prinja, R. K., Barlow, M. J., \& Howarth, I. D. 1990, ApJ, 361, 607

Puls, J., Vink, J. S., \& Najarro, F. 2008, A\&A Rev., 16, 209

Salpeter, E. E. 1955, ApJ, 121, 161

Scalo, J. 1986, in Luminous stars and associations in galaxies, Proc. Symp., Porto-Kheli, Greece, May 26-31, 1985 (Dordrecht: D. Reidel Publishing Co.), 451

Schaerer, D., \& de Koter, A. 1997, A\&A, 322, 598

Schmutz, W., Leitherer, C., \& Gruenwald, R. 1992, PASP, 104, 1164

Smith, D. M. 2004, in The INTEGRAL Universe (ESA Special Publication 552), ed. V. Schönfelder, G. G. Lichti, \& C. Winkler (Noordwijk: ESA), 45

Smith, L. F., \& Maeder, A. 1991, A\&A, 241, 77

Smith, L., Norris, R. P. F., \& Crowther, P. A. 2005, MNRAS, 337, 1309

Tinsley, B. M. 1978, ApJ, 222, 14

Townsley, L. K., Feigelson, E. D., Montmerle, T., et al. 2003, ApJ, 593, 874

Vacca, W. D., Garmany, C. D., \& Shull, J. M. 1996, ApJ, 460, 914

Vanbeveren, D., Van Bever, J., \& Belkus, H., ApJ, 662, 107

Vazquez, G. A., \& Leitherer, C. 2005, ApJ, 621, 695

Vazquez, G. A., Leitherer, C., Schaerer, D., \& Maeder, A. 2007, ApJ, 663, 995

Vink, J. S., de Koter, A., \& Lamers, H. J. G. L. M. 2000, A\&A, 362, 295

Vink, J. S., de Koter, A., \& Lamers, H. J. G. L. M. 2001, A\&A, 369, 574

Wang, W., Harris, M. J., Diehl, R., et al. 2007, A\&A, 469, 1005

Woosley, S. E., \& Weaver, T. A. 1995, ApJS, 101, 181

Woosley, S. E., Langer, N., \& Weaver, T. A. 1995, ApJ, 448, 315 